\title{
Response of Bottle Gourd (Lagenaria siceraria (Mol.) Standl.) to Organic Nutrient Management
}

\author{
D. H. Patel ${ }^{1}$, J. R. Vadodaria ${ }^{1}$, B. M. Nandre ${ }^{2 *}$, Yogesh Pawar ${ }^{3}$, \\ P. A. Sable ${ }^{4}$ and R. G. Solanki ${ }^{1}$ \\ ${ }^{1}$ Department of Vegetable Science, College of Horticulture, Sardarkrushinagar Dantiwada \\ Agricultural University, Jagudan, India \\ ${ }^{2}$ Department of Horticulture, College of Agriculture, Sardarkrushinagar Dantiwada \\ Agricultural University, Tharad, India \\ ${ }^{3}$ Krushi Vigyan Kendra, Sardarkrushinagar Dantiwada Agricultural University, Deesa, India \\ ${ }^{4}$ Krushi Vigyan Kendra, Sardarkrushinagar Dantiwada Agricultural University, \\ Khedbrahma, India \\ *Corresponding author
}

\section{A B S T R A C T}

The experiment entitled, response of bottle gourd (Lagenaria siceraria (Mol.) Standl.) to organic nutrient management, was carried out during summer-2016 at Horticulture

\section{Keywords}

Azospirillium, Bottle gourd, KSM, PSB, Trichoderma viride, Yield

Article Info

Accepted:

20 June 2020

Available Online:

10 July 2020 Instructional Farm, Department of Horticulture, Chimanbhai Patel College of Agriculture, Sardarkrushinagar Dantiwada Agricultural University, Sardarkrushinagar (Gujarat). Total nine treatment combinations of organic nutrient were tested in the Randomized Block Design with three replications. Application of sources of organic nutrients significantly affected growth, yield and quality parameters. Maximum vine length at 60 DAS (392.50 $\mathrm{cm}$ ) was recorded with treatment $50 \%$ FYM $+50 \%$ poultry manure + biofertilizers (Azospirillium+PSB $+\mathrm{KSM})\left(\mathrm{T}_{4}\right)$. Maximum number of branches per plant (3.18), yield per plant $(3.71 \mathrm{~kg})$ and per meter square $(5.19 \mathrm{~kg})$ was found with application of $50 \%$ castor cake $+50 \%$ poultry manure + biofertilizers + Trichoderma viride + neem oil $\left(\mathrm{T}_{9}\right)$. While maximum fruit diameter $(6.39 \mathrm{~cm})$ was recorded in $50 \%$ Neem cake $+50 \%$ Poultry Manure + Biofertilizers (Azospirillium + PSB + KSM) + Trichoderma viride + neem oil $\left(\mathrm{T}_{8}\right)$. Application of organic nutrients significantly affected soil properties like available nitrogen (195.64 kg/ha), phosphorus $(37.20 \mathrm{~kg} / \mathrm{ha})$ and potassium $(226.80 \mathrm{~kg} / \mathrm{ha})$ were found maximum with treatment 50 per cent $\mathrm{N}$ from $\mathrm{FYM}+50$ per cent $\mathrm{N}$ from Poultry Manure + Biofertilizer (Azospirillium + PSB + KSM) + Trichoderma viride + Neem oil $\left(\mathrm{T}_{7}\right)$.

\section{Introduction}

Bottle gourd (Lagenaria siceraria (Mol.) Standl.) belongs to the family cucurbitacece commonly known as Lauki, Kaddu, Ghiya and Dudhi one of the important protective food and supplies of adequate quantities of vitamins, proteins, carbohydrates and 
minerals. Use of organic nutrients and biofertilizers alone or in combination improves efficiency, efficacy of applied fertilizers (Singh and Biswas, 2000), supplies of essential plant nutrients, improve the physicochemical properties, increases water holding capacity, encourages the soil microbial activities thus maintains soil health. Organic nutrient management in bottle gourd by application of nitrogen through FYM, poultry manure, neem and castor cake as potential source of organic matter, application of biofertilizers as Azospirillium, phosphorus solubilizing bacteria (PSB) and potassium solubilizing microorganisms (KSM) ensure the sustainable production, bioagents as Trichoderma viride and neem oil protect the main produce organically from various diseases and pests and enhances growth, yield and marketable quality. The organic manures and bio-fertilizers reduce the leaching loss which resulted in increased availability of nutrients ( $\mathrm{N}, \mathrm{P}$ and $\mathrm{K}$ ) for longer period and improves soil health.

\section{Materials and Methods}

The present investigation entitled, response of bottle gourd (Lagenaria siceraria (Mol.) Standl.) to organic nutrient management was conducted during summer-2016 at Horticulture Instructional Farm, Department of Horticulture, Chimanbhai Patel College of Agriculture, Sardarkrushinagar Dantiwada Agricultural University, Sardarkrushinagar (Gujarat). Experiment was laid out in randomized block design with different combinations of organic nutrients viz. $\mathrm{T}_{1}=$ $50 \%$ FYM $+50 \%$ Poultry Manure $\left(\mathrm{f}_{1}\right), \mathrm{T}_{2}=$ $50 \%$ Neem cake $+50 \%$ Poultry Manure $\left(\mathrm{f}_{2}\right)$, $\mathrm{T}_{3}=50 \%$ Castor cake $+50 \%$ Poultry Manure $\left(\mathrm{f}_{3}\right), \mathrm{T}_{4}=\mathrm{T}_{1}+$ Biofertilizers (Azospirillium $+\mathrm{PSB}+\mathrm{KSM})\left(\mathrm{f}_{4}\right), \mathrm{T}_{5}=\mathrm{T}_{2}+$ Biofertilizers (Azospirillium $+\mathrm{PSB}+\mathrm{KSM})\left(\mathrm{f}_{5}\right), \mathrm{T}_{6}=\mathrm{T}_{3}+$ Biofertilizers (Azospirillium +PSB $+\mathrm{KSM}$ ) $\left(\mathrm{f}_{6}\right), \mathrm{T}_{7}=\mathrm{T}_{4}+$ Trichoderma viride + Neem oil $\left(\mathrm{f}_{7}\right), \mathrm{T}_{8}=\mathrm{T}_{5}+$ Trichoderma viride + Neem oil $\left(\mathrm{f}_{8}\right), \mathrm{T}_{9}=\mathrm{T}_{6}+$ Trichoderma viride + Neem oil $\left(f_{9}\right)$. All the nine treatments were applied at the time of field preparation. Observations were recorded periodically in relation to growth, yield and quality parameters and the mean data were subjected to statistical analysis following analysis of variance technique (Panse and Sukhatme, 1985).

\section{Results and Discussion}

\section{Growth parameters}

The mean data presented in Table 1 showed significant difference for growth parameters among different levels of organic nutrient.

Inspection of data showed significant difference for vine length $(\mathrm{cm})$ at 60 DAS among different levels of sources of organic nutrient. Maximum vine length $(392.50 \mathrm{~cm})$ was recorded with application of $50 \%$ FYM $+50 \%$ poultry manure + biofertilizers (Azospirillium $+\mathrm{PSB}+\mathrm{KSM})\left(\mathrm{T}_{4}\right)$ and it was statistically at par with $\mathrm{T}_{8}$. While, minimum vine length $(334.17 \mathrm{~cm})$ was found in treatment $50 \% \mathrm{FYM}+50 \%$ poultry manure $\left(\mathrm{T}_{1}\right)$. This might be due to the application of organic manure along with biofertilizer is necessary to increase the content of organic matter, improve the physical and chemical properties of the soil and maintain the nutrients balance for crops. This study was supported by Aldal and Hussein (2016) in squash.

Number of branches per plant at 45 DAS was significantly affected by organic nutrient. Maximum number of branches per plant (3.18) was observed with application of $50 \%$ castor cake $+50 \%$ poultry manure + biofertilizers (Azospirillium + PSB + KSM) + Trichoderma viride + neem oil $\left(\mathrm{T}_{9}\right)$ and it was statistically at par with treatment $\mathrm{T}_{7}$. While, minimum number of branches per plant (2.80) 
was observed in treatment $50 \%$ neem cake + $50 \%$ poultry manure + biofertilizers (Azospirillium $+\mathrm{PSB}+\mathrm{KSM})+$ Trichoderma viride + neem oil $\left(\mathrm{T}_{8}\right)$. These might be due to organic manure and biofertilizer improves water holding capacity, availability of nutrient and micro nutrients. This finding is in the accordance with results of Negi et al., (2017) in broccoli and Singh et al., (2015) in cabbage.

In case of growth parameters vine length at 30 DAS and leaf area at 45 DAS was showed numerical differences but organic nutrients can't exert significant deference statistically within them.

\section{Flowering parameters}

Data pertaining to Table 1, organic nutrients can't exert statistically significant deference in flowering parameters viz., days taken for initiation of flowering, number of male and female flower per plant.

\section{Yield parameters}

Data showed in Table 2 revealed that effect of organic nutrients was found non significant with respect to days taken for fruit set to edible harvesting, sex ratio, per cent fruit set, number of fruits per plant and average weight of fruit.

Maximum yield per plant $(3.71 \mathrm{~kg})$ and per meter square $(5.19 \mathrm{~kg})$ was observed with treatment $50 \%$ castor cake $+50 \%$ poultry manure + biofertilizer (Azospirillium + PSB + $\mathrm{KSM})+$ Trichoderma viride + neem oil $\left(\mathrm{T}_{9}\right)$ and it was statistically at par with treatment $\mathrm{T}_{8}$. Whereas, minimum yield per plant $(2.83$ $\mathrm{kg})$ and per meter square $(4.13 \mathrm{~kg})$ was found in treatment $50 \% \mathrm{FYM}+50 \%$ poultry manure $\left(\mathrm{T}_{1}\right)$. These might be due to the sources of organic nutrient favor increase in yield per plant because of favorable soil condition which increase uptake of NPK nutrients due to the influence of biofertilizers which provide favorable conditions around the root rhizosphere resulted in better absorption of nutrients. The results obtained are in line with the findings of Anjanappa et al., (2012) in cucumber.

\section{Quality parameters}

Data (Table 2) showed that effect of sources of organic nutrient was found not significant with respect to fruit length, but fruit diameter was significantly influenced by organic nutrients.

Maximum fruit diameter $(6.39 \mathrm{~cm})$ was recorded in treatment $50 \%$ neem cake $+50 \%$ poultry manure + biofertilizers (Azospirillium + PSB + KSM) + Trichoderma viride + neem oil $\left(\mathrm{T}_{8}\right)$ and it was statistically at par with $\mathrm{T}_{9}$. While minimum fruit diameter $(5.20 \mathrm{~cm})$ was observed with treatment $50 \%$ FYM $+50 \%$ poultry manure $\left(\mathrm{T}_{1}\right)$. This might be due to the sources of organic nutrient favor increase in photosynthesis activity and accumulation of carbohydrates which helps in better fruit growth. These findings are corroborated with the findings of Das et.al (2015) in bottle gourd.

\section{NPK estimation in soil after harvesting (kg/ha)}

The data of Table 3 revealed that nitrogen, phosphorus and potassium estimation in soil after harvesting was significantly influenced by different organic nutrient.

Data clearly indicated that the organic nutrients exerted significant influenced on nitrogen estimation. Significantly higher nitrogen estimation in soil after harvesting $(195.64 \mathrm{~kg} / \mathrm{ha})$ was observed by the treatment $50 \% \mathrm{~N}$ from FYM $+50 \% \mathrm{~N}$ from poultry manure + bio fertilizes (Azospirillium + PSB 
$+\mathrm{KSM})+$ Trichoderma viride + neem oil $\left(\mathrm{T}_{7}\right)$, while lower nitrogen estimation in soil after harvesting $(187.31 \mathrm{~kg} / \mathrm{ha})$ was recorded with treatment $50 \% \mathrm{~N}$ from FYM $+50 \% \mathrm{~N}$ from poultry manure $\left(\mathrm{T}_{1}\right)$. Increase in available $\mathrm{N}$ status of soil after harvest may be due to continuous symbiotic fixation of nitrogen by bio organism through organic acid and reducing the soil $\mathrm{pH}$.

Significantly higher phosphorus estimation in soil after harvesting (37.20 $\mathrm{kg} / \mathrm{ha})$ was observed by the treatment $50 \% \mathrm{~N}$ from FYM $+50 \% \mathrm{~N}$ from poultry manure + bio fertilizes (Azospirillium + PSB + KSM $)+$ Trichoderma viride + neem oil $\left(\mathrm{T}_{7}\right)$. While lower phosphorus estimation in soil after harvesting $(31.63 \mathrm{~kg} / \mathrm{ha})$ was recorded with treatment 50 $\% \mathrm{~N}$ from FYM $+50 \% \mathrm{~N}$ from poultry manure + bio fertilizes (Azospirillium + PSB $+\mathrm{KSM})\left(\mathrm{T}_{4}\right)$. Significantly increased total $\mathrm{P}_{2} \mathrm{O}_{5}$ due to application of bio fertilizer, bio fertilizers increased availability of $\mathrm{P}_{2} \mathrm{O}_{5}$ in soil due to greater solubilization.

Table.1 Effect of organic nutrient management on growth and flowering parameters

\begin{tabular}{|c|c|c|c|c|c|c|c|}
\hline Treatments & $\begin{array}{c}\text { Vine } \\
\text { length } \\
(\mathrm{cm}) \\
30 \text { DAS }\end{array}$ & $\begin{array}{l}\text { Vine } \\
\text { length } \\
(\mathrm{cm}) \\
60 \mathrm{DAS}\end{array}$ & $\begin{array}{l}\text { Number of } \\
\text { branches } \\
\text { per plant } \\
\text { at } 45 \text { DAS }\end{array}$ & $\begin{array}{c}\text { Leaf } \\
\text { area } \\
\left(\mathrm{cm}^{2}\right) \text { at } \\
45 \text { DAS }\end{array}$ & $\begin{array}{l}\text { Days taken } \\
\text { for initiation } \\
\text { of flowering }\end{array}$ & $\begin{array}{l}\text { Number of } \\
\text { male } \\
\text { flower per } \\
\text { plant }\end{array}$ & $\begin{array}{l}\text { Number of } \\
\text { female } \\
\text { flower per } \\
\text { plant }\end{array}$ \\
\hline \multicolumn{8}{|l|}{$\begin{array}{l}\text { Sources of organic nutrient } \\
\text { (F) }\end{array}$} \\
\hline $\begin{array}{l}T_{1}=50 \% \text { FYM }+50 \% \\
\text { Poultry Manure }\left(f_{1}\right)\end{array}$ & 51.00 & 334.17 & 3.03 & 3586.06 & 45.50 & 49.07 & 15.03 \\
\hline $\begin{array}{l}T_{2}=50 \% \text { Neem cake }+ \\
50 \% \text { Poultry Manure }\left(f_{2}\right)\end{array}$ & 52.83 & 367.17 & 2.88 & 3630.71 & 45.17 & 46.57 & 16.50 \\
\hline $\begin{array}{l}T_{3}=50 \% \text { Castor cake }+ \\
50 \% \text { Poultry Manure }\left(f_{3}\right)\end{array}$ & 56.33 & 375.17 & 3.02 & 3615.18 & 45.00 & 47.03 & 17.70 \\
\hline $\begin{array}{l}\mathbf{T}_{4}=\mathrm{T}_{1}+\text { Biofertilizers } \\
(\text { Azospirillium }+\mathrm{PSB}+\mathrm{KSM}) \\
\left(\mathbf{f}_{4}\right)\end{array}$ & 53.17 & 392.50 & 3.13 & 3641.13 & 45.00 & 51.33 & 17.23 \\
\hline $\begin{array}{l}T_{5}=T_{2}+\text { Biofertilizers } \\
(\text { Azospirillium }+ \text { PSB }+ \text { KSM }) \\
\left(\mathbf{f}_{5}\right)\end{array}$ & 51.83 & 388.33 & 3.10 & 3658.75 & 45.83 & 48.83 & 17.17 \\
\hline $\begin{array}{l}\mathrm{T}_{6}=\mathrm{T}_{3}+\text { Biofertilizers } \\
\text { (Azospirillium+PSB+KSM) } \\
\left(\mathrm{f}_{6}\right)\end{array}$ & 53.33 & 358.33 & 3.15 & 3619.62 & 44.00 & 48.43 & 16.90 \\
\hline $\begin{aligned} & \mathbf{T}_{7}=\mathbf{T}_{4}+\text { Trichoderma viride } \\
&+ \text { Neem oil }\left(\mathbf{f}_{7}\right)\end{aligned}$ & 53.67 & 379.67 & 3.15 & 3638.98 & 45.33 & 48.17 & 16.90 \\
\hline $\begin{array}{l}\mathbf{T}_{8}=\mathbf{T}_{5}+\text { Trichoderma } \\
\text { viride }+ \text { Neem oil }\left(\mathbf{f}_{8}\right)\end{array}$ & 54.00 & 390.50 & 2.80 & 3630.60 & 46.17 & 47.00 & 17.57 \\
\hline $\begin{array}{l}T_{9}=T_{6}+\text { Trichoderma } \\
\text { viride }+ \text { Neem oil }\left(f_{9}\right)\end{array}$ & 53.33 & 377.33 & 3.18 & 3613.19 & 47.83 & 49.83 & 17.70 \\
\hline S.Em. \pm & 1.50 & 11.65 & 0.09 & 49.33 & 1.09 & 1.64 & 0.62 \\
\hline C.D. at 5\% & NS & 33.47 & 0.25 & NS & NS & NS & NS \\
\hline C. V.\% & 6.89 & 7.64 & 6.99 & 3.33 & 5.86 & 8.28 & 8.95 \\
\hline
\end{tabular}


Table.2 Effect of organic nutrient management on yield and quality parameters

\begin{tabular}{|c|c|c|c|c|c|c|c|c|c|}
\hline Treatments & $\begin{array}{c}\text { Days taken } \\
\text { for fruit set to } \\
\text { edible harvesting }\end{array}$ & $\begin{array}{l}\text { Sex } \\
\text { ratio }\end{array}$ & $\begin{array}{l}\text { Per cent } \\
\text { fruit set }\end{array}$ & $\begin{array}{c}\text { Number } \\
\text { of fruits } \\
\text { per plant }\end{array}$ & $\begin{array}{l}\text { Average } \\
\text { weight of } \\
\text { fruit }(g)\end{array}$ & $\begin{array}{l}\text { Yield per } \\
\text { plant }(k g)\end{array}$ & $\begin{array}{c}\text { Yield per } \\
\text { meter } \\
\text { square }(\mathbf{k g})\end{array}$ & $\begin{array}{c}\text { Fruit } \\
\text { length } \\
(\mathrm{cm})\end{array}$ & $\begin{array}{c}\text { Fruit } \\
\text { diameter } \\
(\mathrm{cm})\end{array}$ \\
\hline \multicolumn{10}{|l|}{ Sources of organic nutrient $(\mathbf{F})$} \\
\hline $\begin{array}{l}T_{1}=50 \% \text { FYM }+50 \% \text { Poultry } \\
\text { Manure }\left(f_{1}\right)\end{array}$ & 5.92 & 4.03 & 58.03 & 4.60 & 470.50 & 2.83 & 4.13 & 22.27 & 5.20 \\
\hline $\begin{array}{l}T_{2}=50 \% \text { Neem cake }+50 \% \\
\text { Poultry Manure }\left(f_{2}\right)\end{array}$ & 5.98 & 3.99 & 57.43 & 4.56 & 540.67 & 2.20 & 4.45 & 22.34 & 5.64 \\
\hline $\begin{array}{l}T_{3}=50 \% \text { Castor cake }+50 \% \\
\text { Poultry Manure }\left(f_{3}\right)\end{array}$ & 6.00 & 4.07 & 55.66 & 4.61 & 554.50 & 3.22 & 4.42 & 23.33 & 5.94 \\
\hline $\begin{array}{l}\mathbf{T}_{4}=\mathbf{T}_{1}+\text { Biofertilizers } \\
(\text { Azospirillium }+\mathrm{PSB}+\mathrm{KSM})\left(\mathbf{f}_{4}\right)\end{array}$ & 6.10 & 4.01 & 58.23 & 4.30 & 564.67 & 3.24 & 4.44 & 22.59 & 5.51 \\
\hline $\begin{array}{l}T_{5}=T_{2}+\text { Biofertilizers } \\
\left(\text { Azospirillium }+ \text { PSB }+ \text { KSM) }\left(\mathbf{f}_{5}\right)\right.\end{array}$ & 6.07 & 4.01 & 55.73 & 4.60 & 576.33 & 3.20 & 4.66 & 24.04 & 5.63 \\
\hline $\begin{array}{l}T_{6}=T_{3}+\text { Biofertilizers } \\
\left(\text { Azospirillium }+ \text { PSB+KSM) }\left(f_{6}\right)\right.\end{array}$ & 5.88 & 4.26 & 58.97 & 4.46 & 582.67 & 3.20 & 4.61 & 23.46 & 5.81 \\
\hline $\begin{array}{l}\mathbf{T}_{7}=\mathbf{T}_{4}+\text { Trichoderma viride }+ \\
\text { Neem oil }\left(\mathbf{f}_{7}\right)\end{array}$ & 6.12 & 4.05 & 56.90 & 4.73 & 542.83 & 3.02 & 5.00 & 23.38 & 6.13 \\
\hline $\begin{array}{l}T_{8}=T_{5}+\text { Trichoderma viride }+ \\
\text { Neem oil }\left(\mathbf{f}_{8}\right)\end{array}$ & 6.13 & 3.81 & 59.50 & 4.86 & 590.00 & 3.51 & 5.17 & 21.87 & 6.39 \\
\hline $\begin{array}{l}T_{9}=T_{6}+\text { Trichoderma viride }+ \\
\text { Neem oil }\left(\mathbf{f}_{9}\right)\end{array}$ & 6.20 & 4.04 & 60.83 & 4.95 & 575.84 & 3.71 & 5.19 & 22.42 & 6.11 \\
\hline S.Em. \pm & 0.14 & 0.13 & 1.45 & 0.19 & 41.56 & 0.13 & 0.17 & 0.68 & 0.24 \\
\hline C.D. at 5\% & NS & NS & NS & NS & NS & 0.37 & 0.53 & $\mathrm{NS}$ & 0.69 \\
\hline C. V.\% & 5.67 & 7.78 & 6.14 & 9.86 & 18.32 & 9.87 & 9.70 & 7.26 & 10.16 \\
\hline
\end{tabular}


Table.3 Effect of organic nutrient management on available NPK of soil

\begin{tabular}{|c|c|c|c|}
\hline \multirow[t]{4}{*}{ Organic nutrient $(F)$} & $\begin{array}{c}\text { Nitrogen } \\
\text { content } \\
(\mathrm{kg} / \mathrm{ha})\end{array}$ & $\begin{array}{c}\text { Phosphorus } \\
\text { content } \\
\text { (kg/ha) }\end{array}$ & $\begin{array}{c}\text { Potassium } \\
\text { content } \\
\text { (kg/ha) }\end{array}$ \\
\hline & \multicolumn{3}{|c|}{ Before planting } \\
\hline & 180.60 & 29.55 & 200.16 \\
\hline & \multicolumn{3}{|c|}{ After harvesting } \\
\hline $\begin{array}{l}T_{1}=50 \% \mathrm{~N} \text { from FYM }+\mathbf{5 0 \%} \mathrm{N} \text { from Poultry Manure } \\
\left(\mathbf{f}_{1}\right)\end{array}$ & 187.31 & 33.55 & 218.52 \\
\hline $\begin{array}{l}\mathbf{T}_{2}=50 \% \mathrm{~N} \text { from Neem cake }+\mathbf{5 0 \%} \mathrm{N} \text { from Poultry } \\
\text { Manure }\left(\mathrm{f}_{2}\right)\end{array}$ & 188.60 & 34.34 & 218.99 \\
\hline $\begin{array}{l}\mathrm{T}_{3}=50 \% \mathrm{~N} \text { from Castor cake }+50 \% \mathrm{~N} \text { from Poultry } \\
\text { Manure }\left(\mathrm{f}_{3}\right)\end{array}$ & 189.46 & 36.66 & 216.71 \\
\hline $\mathbf{T}_{4}=\mathrm{T}_{1}+$ Biofertilizer (Azospirillium $\left.+\mathrm{PSB}+\mathrm{KSM}\right)\left(\mathbf{f}_{4}\right)$ & 190.72 & 31.63 & 222.16 \\
\hline $\mathbf{T}_{5}=\mathbf{T}_{2}+$ Biofertilizer (Azospirillium $\left.+\mathrm{PSB}+\mathrm{KSM}\right)\left(\mathbf{f}_{5}\right)$ & 194.94 & 33.91 & 217.87 \\
\hline $\mathbf{T}_{6}=\mathrm{T}_{3}+$ Biofertilizer $($ Azospirillium $+\mathrm{PSB}+\mathrm{KSM})\left(\mathbf{f}_{6}\right)$ & 195.62 & 35.31 & 211.32 \\
\hline $\mathbf{T}_{7}=\mathbf{T}_{4}+$ Trichoderma viride + Neem oil $\left(\mathbf{f}_{7}\right)$ & 195.64 & 37.20 & 226.80 \\
\hline $\mathbf{T}_{8}=\mathbf{T}_{5}+$ Trichoderma viride + Neem oil $\left(\mathbf{f}_{8}\right)$ & 195.51 & 34.88 & 212.91 \\
\hline $\mathbf{T}_{9}=\mathbf{T}_{6}+$ Trichoderma viride + Neem oil $\left(\mathbf{f}_{9}\right)$ & 194.58 & 35.10 & 212.18 \\
\hline S.Em. \pm & 2.20 & 0.49 & 3.29 \\
\hline C.D. at 5\% & 6.32 & 1.40 & 9.47 \\
\hline C. V.\% & 2.80 & 3.45 & 3.71 \\
\hline
\end{tabular}

Significantly higher potassium estimation in soil after harvesting (226.80 $\mathrm{kg} / \mathrm{ha})$ was observed by the treatment $50 \% \mathrm{~N}$ from FYM $+50 \% \mathrm{~N}$ from poultry manure + bio fertilizes $($ Azospirillium + PSB + KSM $)+$ Trichoderma viride + neem oil $\left(\mathrm{T}_{7}\right)$.

While lower potassium estimation in soil after harvesting $(211.32 \mathrm{~kg} / \mathrm{ha})$ was recorded with treatment $50 \% \mathrm{~N}$ from caster cake $+50 \% \mathrm{~N}$ from poultry manure + bio fertilizes (Azospirillium +PSB+ KSM) $\left(\mathrm{T}_{6}\right)$. This may be due to application of bio fertilizer increase availability of potassium in soil.

To achieve better growth and maximum yield, bottle gourd should apply 50\% $\mathrm{N}$ from castor cake $+50 \% \mathrm{~N}$ from poultry manure + biofertilizers (Azospirillium+PSB+KSM) + Trichoderma viride + neem oil.

\section{References}

Aldal, H. K. and Hussein A. H. (2016). Effect of NPK (Cucurbita pepo L.) and chicken manure on the productivity and some growth components of squash. ARPN Journal of Agricultural and Biological Science. 11(6): 230-235.

Anjanappa, M.; Venkatesha, J. and Kumara, B. S. (2012). Growth, Yield and quality attributes cucumber (cv. Hasan local) as influenced by integrated nutrient management grown under protected condition. Vegetable Science. 39 (1): 4750.

Das, R., Mandal, A. R., Priya, Anju., S. P. Das and Kabiraj, J. (2015). Evaluation of integrated management on the performance of the bottle gourd (Lagenaria siceraria (Mol.) Standl.). Journal of Applied and Natural Science. 7(1):18-25. 
Negi, Ekta.,Punetha, Shailaja., Pant, S.C., Kumar, Sandeep., Bahuguna, Pankaj., Mekap, Bengia and Nautiyal, B. P. (2017). Effect of organic manures and bio-fertilize on growth, yield, quality and economics of broccoli (Brassica oleracea L.) var. (Italica plenck) cv. Green head under high-hill conditions of Uttarakhand. International Journal of biological science research.7 (1): 96-100.

Panse, V. G. and Sukhatme, P. V. (1978). Statistical Methods for Agricultural Workers ICAR. Publ., New Delhi. pp. 369.
Singh, G.B. and Biswas, B.P. (2000) Balanced and integrated nutrient management for sustainable crop production. Limitation and future strategies. Fertilizer News, 45: 55-60.

Singh, V. K., Shree, S., Kumar, R., Singh P. and Singh, R. G. (2015). Effect of microbial inoculants and inorganic fertilizers on growth and yield of hybrid cabbage (Brassica oleracea L. Var.capitata). The Bioscan, 10(3): 12271231.

\section{How to cite this article:}

Patel, D. H., J. R. Vadodaria, B. M. Nandre, Yogesh Pawar, P. A. Sable and Solanki, R. G. 2020. Response of Bottle Gourd (Lagenaria siceraria (Mol.) Standl.) to Organic Nutrient Management. Int.J.Curr.Microbiol.App.Sci. 9(07): 2481-2487. doi: https://doi.org/10.20546/ijcmas.2020.907.291 